\title{
Persistent Upward Gaze in Coma and its Prognostic Implications
}

The Editor,

Sir,

Coma due to global anoxia of the brain most commonly following cardiopulmonary arrest continue to be one of the subject of intense scrutiny with recovery said to range from $20-30 \%$ in patients after 24 hours (1).

Factors associated with poor outcome after hypoxia induced coma continue to be extensively studied however literature is rather sparse regarding the prognostic importance of sustained vertical gaze. In one cohort of 50 patients with hypoxic induced coma, the authors described the commencement of upward gaze and suggested that downward gaze was associated with vegetative state in almost half of the patients that they studied (2). We present our experience with four patients who we followed up after hypoxia-induced coma.

The requisite Institutional Review Board approval at McLaren Medical Center was obtained. A wavier of informed consent was obtained with the justification that the research activities present no greater than minimal risk of harm to subjects and involves no procedures for which written consent is normally required outside of the research context. 
Study participants were restricted to adults more than 18 years of age and we used a Glasgow Coma Scale of less than five. We reviewed patients with ages 51,58,73 and 89 years old respectively. We conducted the daily neuroophthalmological examination with specific attention to pupils, spontaneous eye movements and reflex eye movements. Examiners who were trained in evaluating eye gaze reviewed the patients individually and each was blinded to the other examiner's assessment. All the patients in our series had CT, EEG performed, the tendon response was elevated and all the patients remained comatose with no sign of arousal elicited. EEG responses were indicative of moderate encephalopathy of non-specific etiology while CT appeared normal with no evidence of acute damage.

The pattern of ocular deviations did not follow any particular pattern however; we noticed that the appearance of vertical eye deviation was associated with invariable poor prognosis.

Previous authors like Keane have reported sustained vertical upward gaze inpatient with cardiac arrest. $(3,4,5)$ The presence of minimal literature regarding this phenomenon suggests that Investigators have been reluctant to perform more studies to unravel this interesting finding. Our findings suggest that the appearance of the sustained upward gaze invariably heralds the downward spiral of the patient's clinical outcome with the patient dying.

We postulate that this sign be used in addition to other clinical parameters to establish a prognostic timeline for patient. This will enable Neurologists and patient family to appropriately modulate their expectations regarding patient final outcome 
especially in resource challenged areas where there is paucity of life support machines.

Keywords: Coma, persistent upgaze, sunrise sign, upward gaze sign

F Osuagwu ${ }^{1}$, D Pasupuleti ${ }^{1,2}$, RH Bradley $^{1}$

From: ${ }^{1}$ Department of Psychiatry, Central Michigan University College of Medicine, Saginaw, MI USA, ${ }^{2}$ Department of Medicine' Michigan State University, East Lansing, MI, USA.

Correspondence: Dr F Osuagwu, Department of Psychiatry, Central Michigan University College of Medicine, Saginaw, MI USA. 1000 Houghton Avenue, Saginaw, MI 48602.

E-mail:ferdnand.osuagwu@cmich.edu 


\section{REFERENCES}

1. Zandbergen EGI. Postanoxix coma: how (long) should we treat? Eur J Anaesthesiol Suppl 2008; 42: 39-42.

2. Johkura K, A Komiyama and Y Kuroiwa. Vertical conjugate eye deviation in Postresuscitation coma. Annals of neurology.2004: 56: 878-81.

3. Keane JR. Sustained upgaze in coma. Annals of Neurology. 1981: 9: 409-12.

4. Nakada T, Kwee IL and Lee H. Sustained upgaze in coma. Journal of Neuro-Ophthalmology.1984: 4: 35-7.

5. Johkura K, Komiyama A Kuroriwa. Sustained upward gaze in coma after cardiac Arrest. J Neurol Neurosurg Psychiatry. 2001; 71: 278-9. 